\title{
ANALYSIS OF STRESS AND STRAIN TO DETERMINE THE PRESSURE CHANGES IN TIGHT-FITTING GARMENT
}

\author{
Nareerut Jariyapunya and Blažena Musilová \\ Technical University of Liberec, Department of Clothing Technology, Liberec, Czech Republic, \\ Studentská 2, 1402/2, Husova, 46117 Liberec, Czech Republic \\ E-mail: nareerut.j@en.rmutt.ac.th, blazena.musilova@tul.cz
}

\begin{abstract}
:
Based on the mechanical properties of stretch fabrics and Laplace's law, the mathematical models have been developed enabling one to determine the values of the relationship between the fabric strain and the circumferential stress depending on pressure and diameter of the body. The results obtained refer to the values of the parameters assessed for the initial phase of their exploitation, which allow us to preliminarily predict the values of these parameters.
\end{abstract}

\section{Keywords:}

Stretch fabric, circumferential stress, Laplace's law, tight fitting, pressure, elastic

\section{Nomenclature}

$A$, area of the fabric, $\mathrm{m}^{2}$

$a$, cross sectional area of the fabric, $\mathrm{m}^{2}$

$C$, circumference of the cylindrical model, $\mathrm{m}$

$C_{\mathbf{0}}$, initial circumference of the fabric, $\mathrm{m}$

$D$, diameter of the cylindrical model, $\mathrm{m}$

$F$, force of the fabric stretched, N

$\boldsymbol{h}$, thickness of the fabric, $m$

$P$, pressure, $\mathrm{Pa}$

$R$, radius of the cylindrical model, $\mathrm{m}$

$T$, tension of the fabric, $\mathrm{N} / \mathrm{m}$

$w$, width of the fabric, $m$

$\sigma$, stress in the fabric, $\mathrm{Pa}$

$\varepsilon$, strain of the fabric

$\varphi$, tangent of the cylindrical, degree

\section{Introduction}

Tight-fitting garments are considered as special pressure garments that have been widely researched and utilized in the fields of medical application, athletic application and body shaping applications [1]. Regarding production procedures of tight-fitting garment, it is rather complicated and difficult to achieve specific pressure depending on the aforementioned application functions. To develop the method for customizing pressure garment, there are three main steps that should always be carried out. The first step is body measurement, which is a beginning for achieving the actual size of the human body. The second phase is to investigate a mathematical model to determine the size of pattern construction based on Laplace's law theory and characteristic of fabric properties of tight-fitting garment and pressure exerted [2], and the last step is to develop a pattern construction of tight-fitting garment based on the mathematic model [3, 4].

Concerning the tight-fitting garment, is generally applied to develop patterns, and the construction of patterns are optimized from the perspective of the stretch ability of elastomeric fabrics $[5,6]$ and the influential of pattern construction that affects production of tight-fitting garment is pressure garment $[2,7,8]$. However, the exact pressure garment required for effective treatment has never been scientifically established [9-12] unless in the case of the pressure comfortable for tightfitting garment, Makabe [13] found that the human body will have uncomfortable perception when the pressure is $>4.00$ $5.33 \mathrm{kPa}$. Moreover, Makabe [14] and Makabe et al [15] have observed that the pressure in waist impacts covering area, breathing and movement in the medium range of garment pressure $1.47-2.45 \mathrm{kPa}$. Liu et al [16] presented that the comfortable pressure range on breast was converted into bust girth range and was found to be $0.96-1.35 \mathrm{kPa}$. Moreover, Ito [17] presented that the pressure range for the most comfortable compression feeling in different parts of the body should be less than $1.27 \mathrm{kPa}$.

There are various theories that assist predicting pressure value of the clothing worn on the body; however, countless numbers 
of researchers commonly choose and apply Laplace's law as a way to predict pressure of clothing on the human body. In those studies, the basic geometric form of the cylinder is used to represent human figure [18-21]. Kowalski et al. [20] designed the modeling of compression garments by applying Laplace's law with the intended value of unit pressure for the body circumference of a variable curvature radius. Moreover, they also analyzed the characteristics of knitted vascular prostheses to predict the modeling for the determination of circumferential longitudinal force depending on the prosthesis pressure and diameter [21]. Furthermore, Leung et al. [22] stated that rather than using discrete experimental tension data Laplace's law can be applied to predict the pressure model for compression garment assisted by using fabric stiffness values that are mathematically calculated from stress and strain curve.

Interestingly, many researchers aimed to study the pressure garment in order to predict the pressure value of clothing, whereas the tight-fitting garment was investigated to find out the way in order to achieve specific pressure by reducing the size of circumference of clothing. Novelty of this research article is that the work will be focusing on the prediction of the mathematical model of strain value of fabric stretched by determining the specific pressure and the body circumference. In this way, initial fabric circumference could be calculated and be applied to determine the size of pressure garments.

Moreover, the aim of this research article was to describe and analyze the pressure of tight-fitting garment when the fabric was extended when pulled by force while wearing it on the body. Furthermore, analysis of pressure of tight-fitting garment was done by applying Laplace's law theory in order to describe and use stress value instead of the force of fabric extended to predict the modeling. At the final stage, results of fabric stress from the experiment which has a relationship with strain from the stress-strain curve that could be calculated the initial circumference of fabric to assist pattern construction for the tight-fitting garments.

\section{Experimental}

\subsection{Materials}

The materials used in this experimental study were constant fiber composition and knitting machine. A four-track single jersey machine of GOANG LIH, model GLF/s-3F-4T, CYL.DIA 32", GAUGE N.P.I. 28 G, Feeders $96 \mathrm{~F}$ was run at a constant speed of $22 \mathrm{rpm}$. The characteristics of elastic knitted fabrics used in the study are presented in Table 1.

\subsection{Methods}

\section{Tensile testing}

In the experimental study, the mechanical characteristics of knitted fabric are taken into account including the relationship between force-elongation values and the assumption of those values that are at the steady state in the fifth cycle of stretching and decompressing process. The experiment was tested based on the standard EN 14704-1 strip test (determination of elasticity of fabric strip test) [23], and the specimens were $250 \mathrm{~mm}$ in length, $50 \mathrm{~mm}$ in width and the gauge length was $100 \mathrm{~mm}$. The constant rate extension (CRE) and retraction rate was $500 \mathrm{~mm} / \mathrm{min}$, which determined the extension to be $50 \%$ at the fifth cycle testing to obtain repeatable stress-strain curves.

\section{Data analysis of Laplace's law}

The aim at this part of the experiment is to describe the compression pressure of pressure garment at which the fabric was extended while wearing on the body by applying Laplace's law theory.

The following assumptions were accepted in the considerations:

1. The relationship between the pressure of tight-fitting garment and force of the fabric extended is described by Laplace's Law as shown in equation (1).

2. The experiment determined fabric extension in a cylindrical shape as it is similar to human body shape.

3. The circumferential stress or hoop stress is the main focus of the testing; therefore, the ratio of thickness to inside diameter of the cylinder with very less can be neglected.

4. The relation between stress-strain of elastic fabrics will be determined on the basis of experimental characteristics for the stress-strain phase of the stretch fabric in five hysteresis cycles.

Table 1. Elastic knitted fabrics' characteristics.

\begin{tabular}{|l|c|c|c|c|c|c|}
\hline \multirow{2}{*}{ Parameters } & \multicolumn{5}{|c|}{ Single jersey knitted fabrics } \\
\cline { 2 - 7 } & $\mathbf{4 0 2}$ & $\mathbf{4 0 3}$ & $\mathbf{4 0 7}$ & $\mathbf{7 0 2}$ & $\mathbf{7 0 3}$ & $\mathbf{7 0 7}$ \\
\hline Polyamide (dtex/ply) & $44 / 2$ & $44 / 2$ & $44 / 2$ & $78 / 2$ & $78 / 2$ & $78 / 2$ \\
\hline Elastane (dtex/ply) & $22 / 1$ & $33 / 1$ & $78 / 1$ & $22 / 1$ & $33 / 1$ & $78 / 1$ \\
\hline Weight (g/m $\left.{ }^{2}\right)$ & 215.24 & 218.17 & 260.48 & 270.87 & 305.14 & 318.49 \\
\hline Thickness (mm) & 0.55 & 0.55 & 0.50 & 0.60 & 0.61 & 0.57 \\
\hline Polyamide (PA)\% & 90.79 & 87.59 & 74.52 & 94.38 & 92.94 & 83.46 \\
\hline Elastane (E)\% & 9.21 & 12.41 & 25.48 & 5.62 & 7.06 & 16.54 \\
\hline
\end{tabular}


By applying Laplace's law, the determination of pressure value requirement for the prediction of strain value of the stretch fabric was conducted. In the testing, the geometric form of the cylinder is assumed to represent human body figure. Figure 1a illustrates the fabric stretch on areas of $(A, B, C, D)$ shown in the cylindrical model, and in Figure $1 \mathrm{~b}$, contact pressure was demonstrated on the cylinder with a small element of fabric stretched from position $A$ to $B$. The force pulls have occurred on fabric at both sides, but curvature of the cylinder surface has created each of these forces unparalleled to the tangent plane. In one direction, force, $F$, acts with two nearly opposite forces of magnitude, each forming a tiny angle of magnitude, $F \sin \frac{d \varphi}{2}$, with the tangent to the surface in one direction. Projecting the total force in the direction of the center of curvature $d F=2 F \sin \frac{d \varphi}{2}=F d \varphi$, where $d A=R d \varphi w$ is the area of the rectangle $(A, B, C, D)$.

Pressure, $P$, is defined as a measure of the force applied over a unit area. For example, fabric acts at the small area of the rectangle element in Figure $1 \mathrm{a}$ and $\mathrm{b}$. Finally, by adding the contribution from the two directions we arrive at Laplace's law for the pressure discontinuity due to force of fabric stretched. It could be observed when dealing with surface tension of stretch fabric that application of Laplace's law is widely taken part in pressure prediction.

$$
P=\frac{d F}{d A}=\frac{F d \varphi}{R d \varphi w}=\frac{F}{R w}=\frac{T w}{R w}=\frac{T}{R}
$$

In the case of considering whole area of the cylindrical model, it could be assumed that $\varphi=360^{\circ}$ or $2 \pi$, as found in the following equation:

$P=\frac{d F}{d A}=\frac{F d \varphi}{R d \varphi w}=\frac{F 2 \pi}{R 2 \pi w}=\frac{T w 2 \pi}{C w}=\frac{T 2 \pi}{C}$

The tension acting on the fabric stretched by the Instron tensile strength machine in Figure 2a illustrates the loading force $F$ and tension $T$ acting on the fabric, and Figure $2 \mathrm{~b}$ represents force $F$ and tension $T$ acting on the fabric on the cylindrical model. In fact, when fabric extended in the length direction then the other direction will be shorter in width direction as shown in Figure $2 a$ and $b$. Because of the experiment, fabric was extended with only $50 \%$ elongation and that would have very a

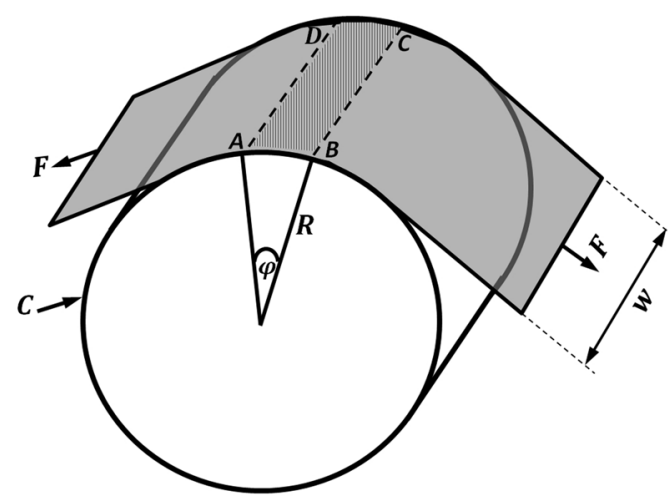

b

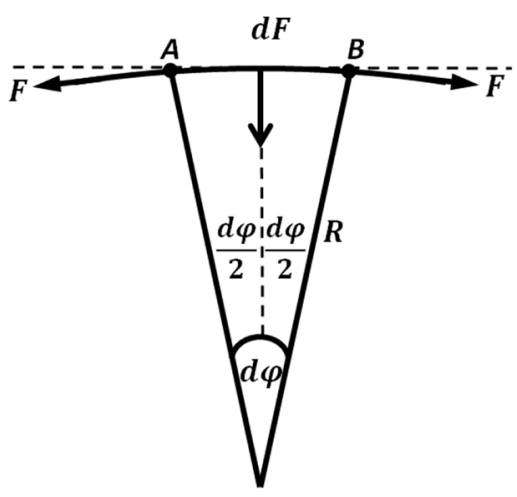

Figure 1. Fabric stretch showing the force directions on the cylindrical model: (a) fabric stretch on the cylindrical model and (b) the pressure contact on surface area.

a

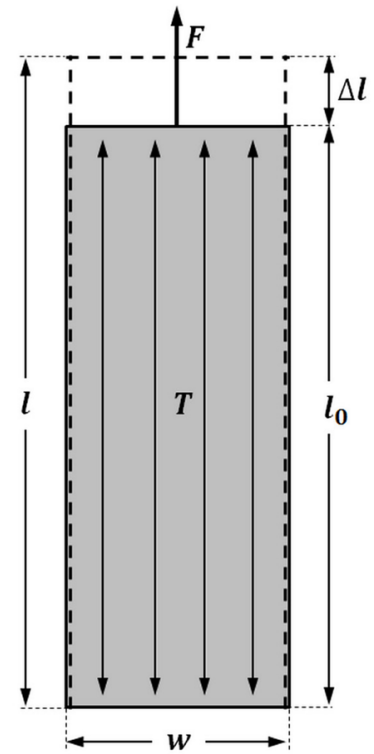

b

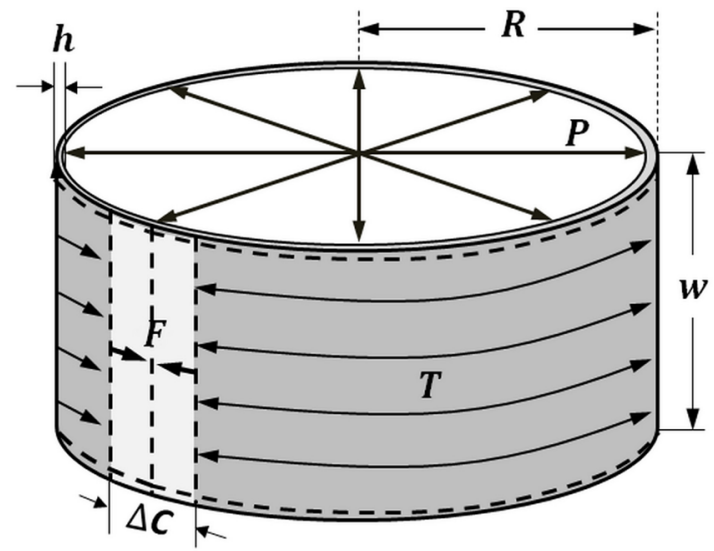

Figure 2. The tension acting on stretch fabric: (a) fabric tension acting on the tensile strength machine and (b) fabric tension acting on the cylindrical model. 
low effect on the decrease in width and therefore it could be assumed that the width after extending the fabric is almost the same as the initial one.

Stress, $\sigma$, is the ratio of force applied to a cross section area and is defined as the force per unit area of a material.

$$
\sigma=\frac{F}{a}=\frac{T w}{h w}=\frac{T}{h}
$$

The tension, $T=\sigma \boldsymbol{h}$, from equation (3) is applied in equation (2) as follows:

$$
P=\frac{\sigma h 2 \pi}{C}
$$

Then, it can be applied for calculating the stress, $\sigma$, related to the fabric which was extended by pulling force, and it is called the circumferential stress [24].

$$
\sigma=\frac{D P}{2 h}
$$

The strain is applied in the cylindrical model to calculate the initial circumference of fabric.

$$
C_{0}=\frac{C}{\varepsilon+1}
$$

\section{Prediction modeling of the fabric strain}

The stress-strain data points were fitted with a third-order polynomial as given in equation (7), which is accurate enough to predict the mechanical characteristics of stretch fabrics between the predicted strain $\varepsilon$ at the magnitude of the fabric stress $\sigma$ based on standard EN 14704-1 [23]. The equation, it can be described with the following the model for prediction equation (7).

$$
\varepsilon(\sigma)=a_{1} \sigma^{3}+a_{2} \sigma^{2}+a_{3} \sigma
$$

By substituting equation (5) of fabric stress in equation (7), equation (8) of the strain value as a function prediction model of its diameter of the cylindrical model, pressure, fabric thickness and mechanical characteristics of stretch fabric (leading coefficients $a_{1}, a_{2}$ and $a_{3}$ ) is obtained.

$$
\varepsilon=a_{1}\left(\frac{D P}{2 h}\right)^{3}+a_{2}\left(\frac{D P}{2 h}\right)^{2}+a_{3}\left(\frac{D P}{2 h}\right)
$$

\section{Results and discussion}

Figure 3 illustrates one example of the hysteresis loop result of the sample 407 , and obviously, the force value at the first loading is highest with a value of $7.68 \mathrm{~N}$. While considering the force value at the fifth cycle decreased to $10.30 \%$ at $6.89 \mathrm{~N}$, it can be observed that the force values are considerably stable and dropped only $0.14 \%$ from that at the loading fourth cycle. The results show that tensile of elastic fabrics changed as a result of low-cycle fatigue (LCF) due to cyclic load of the highest force values that only holds for the initial stage. After a number of cycles, the elastic fabric behaves viscoelastically, and values of force are lower than the first cycle. In this research, the results of force values at the fifth cycle will be considered to calculate the stress-strain curve.

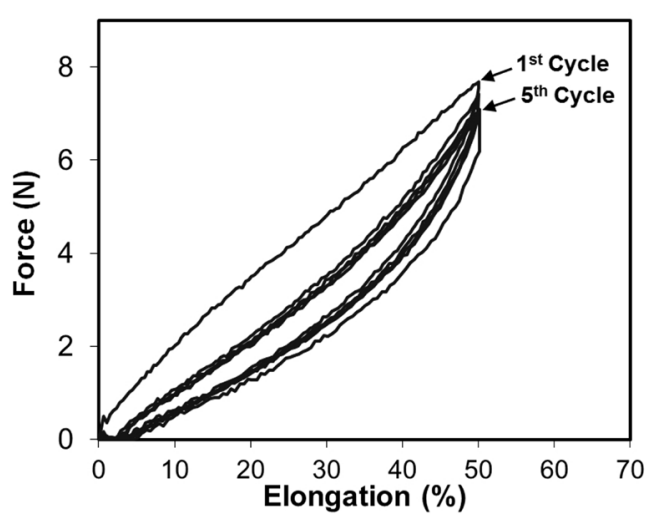

Figure 3. The tensile behavior of elastic fabric after five hysteresis loops.

In the stress-strain curve, the prediction equations of the lines used non-linear functions to predict strain value as shown in Figure 4. Evidently, sample 707 displayed results with highest stress value that could reach $500 \mathrm{kPa}$ at 0.5 of strain value, and therefore, the sample 707 is applicable for use in tightfitting garment. On the other hand, the results of the samples 402 and 403 possessed lesser stress values of $100 \mathrm{kPa}$ at the strain 0.5 , and hence, these fabrics are inappropriate for the use in tight-fitting garment particularly when they could cause a decrease in the size of pattern construction.

At this point of the experiment, the analysis of modeling results of the strain value of stretch fabric depending on their

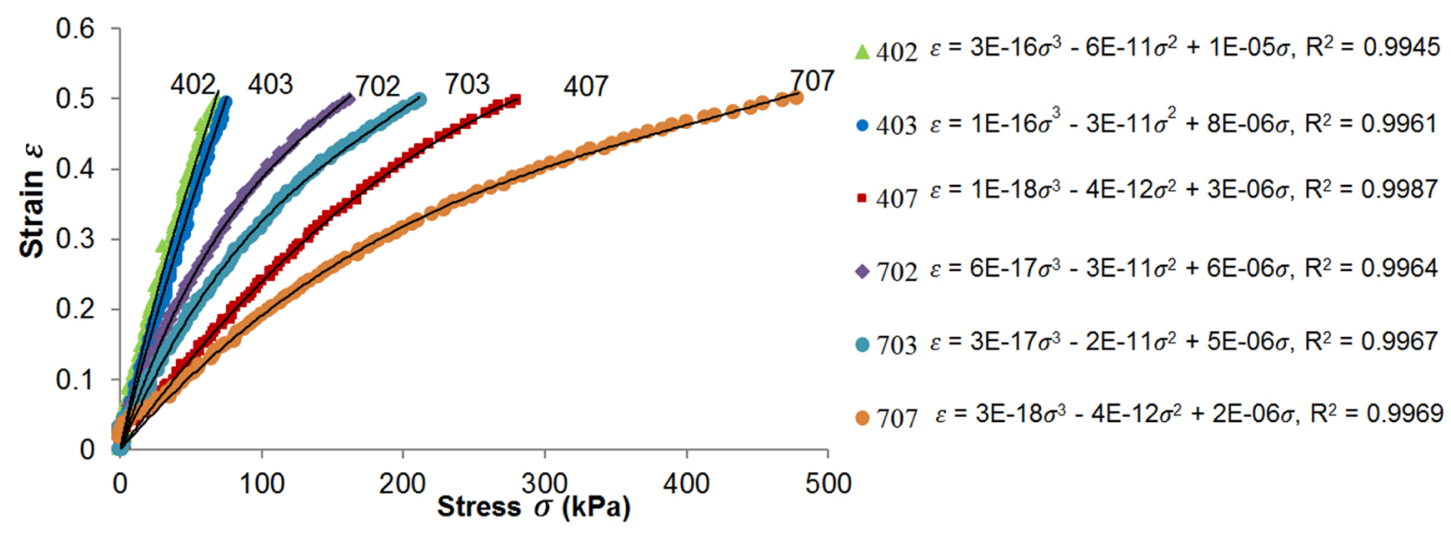

Figure 4. The mechanical characteristics of elastic knitted fabric between stress and strain. 
a

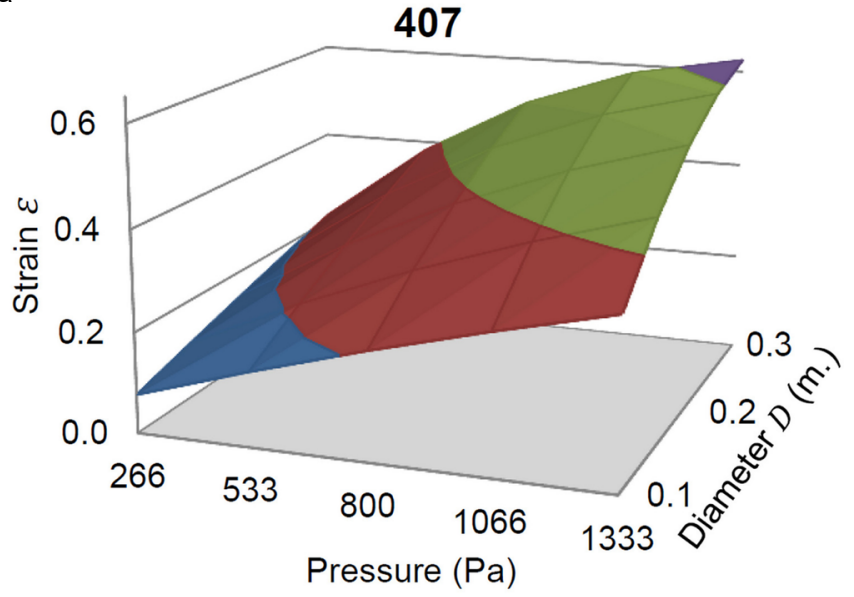

$\square 0.0-0.2 \quad \square 0.2-0.4 \quad \square 0.4-0.6 \quad \square 0.6-0.7$

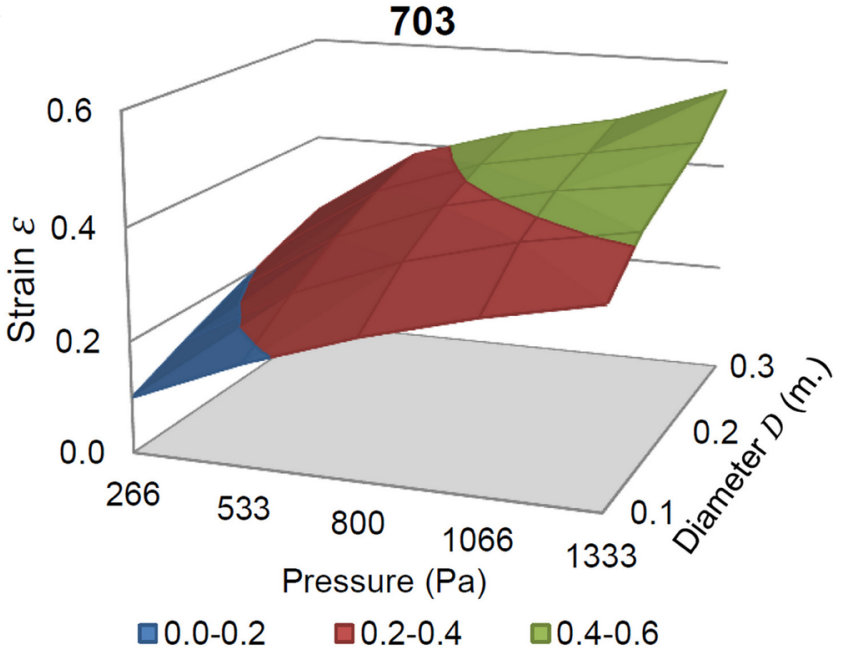

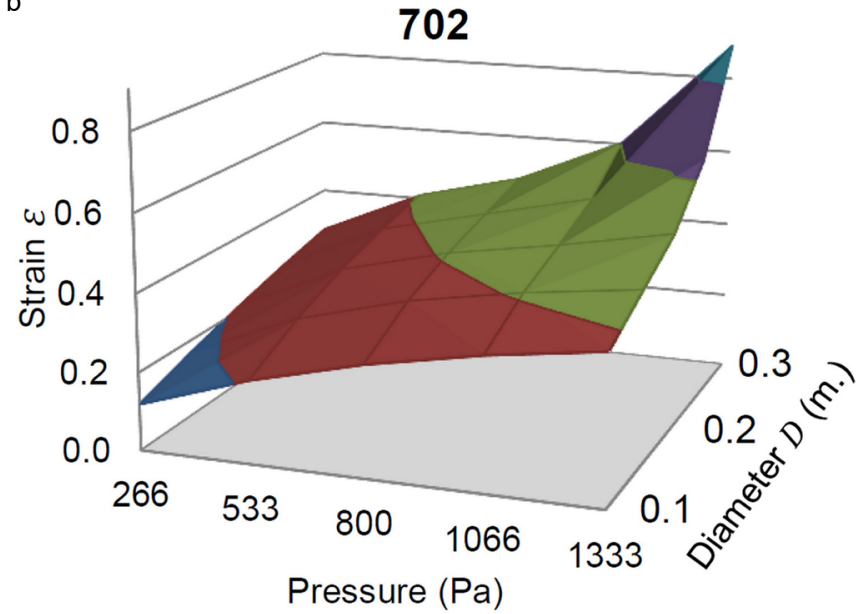

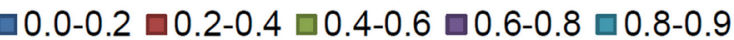

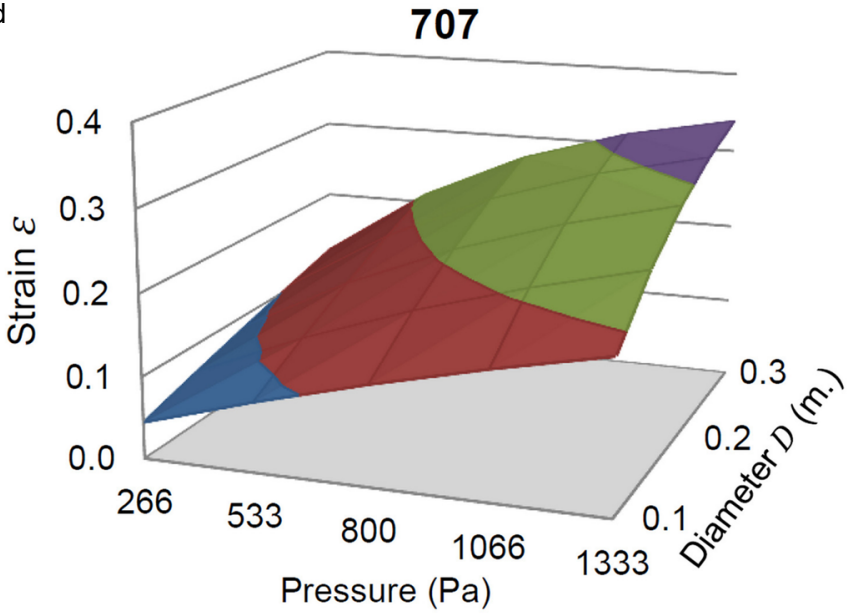

$\square 0.0-0.1 \quad \square 0.1-0.2 \quad \square 0.2-0.3 \quad \square 0.3-0.4$

Figure 5. The stretch fabric effect of pressure and diameter of the body on the strain.

pressure and diameter of the cylindrical model obtained by equation (8) was conducted. The results show that the fabric samples 407, 702, 703, and 707 owing to their stretch fabric properties possessed high stress values that were applicable for producing tight-fitting garment.

The prediction modeling according to the results of stress-strain curves and Laplace's law represents the graphs of predicted strain value depending on pressure and diameter as shown in Figure 5a, b, c and d. It could be found that the strain value was increased corresponding to the diameter and pressure. The graph in Figure 5a illustrated the predicted strain graph of the 407 sample, and the strain values were predicted to be highest around 0.6-0.7. Furthermore, the samples 702, 703 and 707 represented in Figure 5b, $c$ and d illustrated that the predicted strain values had the highest range to be approximately 0.8 $0.9,0.4-0.6$ and $0.3-0.4$, respectively.

In the final part of this research, the strain values for making pattern construction for tight-fitting garment are to be predicted. The experiment was conducted by using the ASTM D5585-95 [25] with the standard sizing system for adult female figure size 8 to predict the strain value. Besides, for this standard size 8 , figures were used for calculating bust girth $89.00 \mathrm{~cm}$ and waist

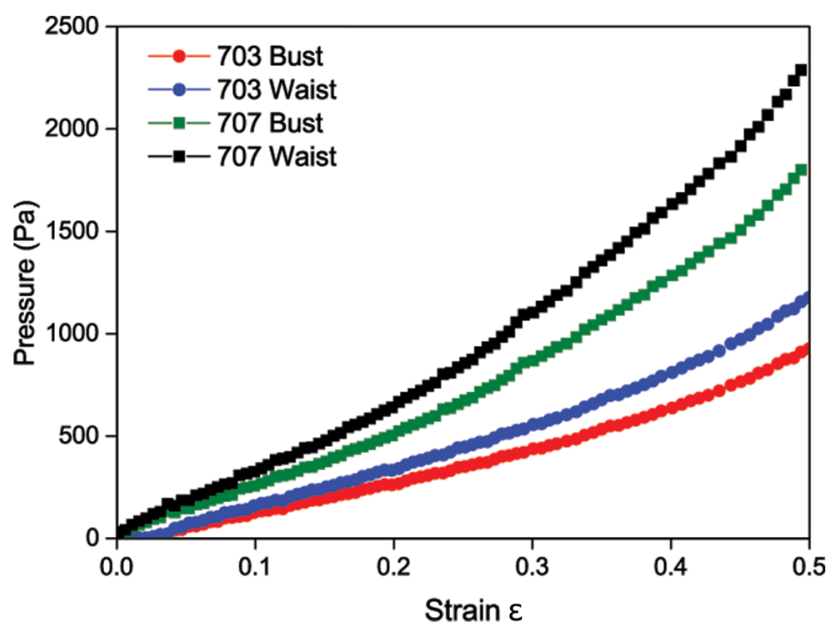

Figure 6. The graph relationship between pressure value and predicted strain value of elastic fabric.

girth $68.50 \mathrm{~cm}$. In Figure 6, the graph illustrates a relationship between pressure values and predicted strain values. The results could be discussed in accordance to different tensile behaviors, different parts of body circumference and ways to apply strain results to create the sizing of pattern construction. 
According to tensile behavior, in Figure 6 it could be found that fabric had high stress that was related to high pressure value. While the extension of fabric had lower strain value as it can be seen from the sample between 703 and 707.

Regarding the different parts of body circumference, it was found that with same condition of fabric sample and same pressure value, fabric had smaller body circumference when the strain value is lower.

At the final stage, prediction modeling was applied to find out the size of pattern construction for tight-fitting garment. From the results of strain value, the initial circumference of fabric $C_{\mathbf{0}}$ can be calculated by equation (6) as mentioned earlier. This method could be guided and made the pattern construction for the tight-fitting garment with specific pressure value requirements.

\section{CONCLUSION}

From the results of analysis based on the mechanical characteristics of stretch fabrics and Laplace's law, it could be concluded that first tensile behavior of elastic from the hysteresis loops test of the force of elastic fabrics could be changed as a result of LCF, and therefore, the results of the tensile test should be considered for its suitable force values for application. Second, the predictive modeling based on the fabric properties from stress-strain curve and Laplace's law theory was considered useful for application to help predict strain values depending on the value of pressure and diameter. Third, the application of predictive modeling for tightfitting garments was used for describing the method when the clothing needs precise compression pressure values. Last but not least, results of stress-strain characteristics could be predicted to find out strain value which was more proximate to the real condition, and this strain value was calculated to get the size of pattern construction of tight-fitting garments. However, it is necessary to examine the fabric properties that are suitable to apply pressure on clothing.

\section{ACKNOWLEDGEMENTS}

This work was supported by the Ministry of Industry and Trade of the Czech Republic, Programme Trio - project "Senior Tex - Smart Modular Clothing and Textile Products with Integrated Electronic Microsystems for Improving the Health Care of the Aging Population and Handicap People", reg. no. FV10111.

\section{References}

[1] Wang, L., Felder, M., Cai, J. (2011). Study of properties of medical compression garment fabrics. Journal of Fiber Bioengineering and Informatics, 4, 15-22.

[2] Jariyapunya, N., Musilová, B., Geršak, J., Baheti, S. (2017). The influence of stretch fabric mechanical properties on clothing pressure. Vlakna a Textil, 24(2), 43-48.

[3] Ying, X., Tao, X. (2018). Compression garments for medical therapy and sports. Polymers, 10(663), 3-19.
[4] Musilová, B. (2012). Predikce konstrukčních parametrů střihů korzetových výrobků. Liberec: Technická univerzita $\checkmark$ Liberci, Fakulta textilní, Katedra oděvnictví.

[5] Ziegert, B., Keil, G. (1998). Stretch fabric interaction with action wearables: defining a body contour pattern system. Clothing and Textile Research Journal, 6, 55-64.

[6] Watkins, P. (2011). Designing with stretch fabrics. Indian Journal of Fibre \& Textile Research, 36, 366-379.

[7] Kilinc-Balci, F.S. (2011). Improving comfort in clothing. Cambridge, Woodhead Publishing.

[8] Liu, Y., Dongsheng, C. (2015). An analysis on EEG power spectrum under pressure of girdle. International Journal of Clothing Science and Technology, 27(4), 495-505.

[9] Cheng, J., Evans, J., Leung, K., Clark, J., Choy, T., et al. (1984). Pressure therapy in the treatment of post-burn hypertrophic scar - a critical look into its usefulness and fallacies by pressure monitoring. Burns, 10, 154-63.

[10] Giele, H., Liddiard, K., Currie, K., Wood, F. (1997). Direct measurement of cutaneous pressures generated by pressure garments. Burns, 23(2), 137-141.

[11] Giele, H., Liddiard, K., Booth, K., Wood, K. (1998). Anatomical variations in pressures generated by pressure garments. Plastic and Reconstructive Surgery, 101(2), 339-406.

[12] Macintyre, L., Baird, M. (2006). Pressure garments for use in the treatment of hypertrophic scars - a review of the problems associated with their use. Burns, 32, 10-15.

[13] Makabe, H. (1991). A study of clothing pressure developed by the girdle. Journal of the Japan Research Association for Textile End-Uses, 3(9), 424-438.

[14] Makabe, H., Momana, H., Mitsuno, T., Ueda, K. (1993). Effect of covered area at the waist on clothing pressure. Sen-iGakkaishi, 49, 513-521.

[15] Mengna, G., Kuzmichev, V. E. (2013). Pressure and comfort perception in the system "female body-dress". AUTEX Research Journal, 13(3), 71-78.

[16] Liu, H., Chen, D., Wei, Q., Panc, R. (2013). An investigation into the bust girth range of pressure comfort garment based on elastic sports vest. The Journal of the Textile Institute, 104(2), 223-230.

[17] Ito, N. (1995). Pressure sensation (clothing pressure) - for the design of ideal clothing. Journal of Japan Research Association for Textile End-Uses, 36(1), 38-43.

[18] Lim, N.-Y., Ng, S.-P., Yu, W., Fan, J. (2006). Innovation and technology of women's intimate apparel. In Pressure evaluation of body shapers (pp. 151-170). Cambridge, UK, Woodhead Publishing Limited.

[19] Zhao, L., Li, X., Yu, J., Li, C., Li, G. (2017). Compression sleeves design based on Laplace Laws. Journal of Textile Engineering \& Fashion Technology, 2(2).

[20] Kowalski, K., Mielicka, E., Kowalski, T. M. (2012). Modelling and designing compression garments with unit pressure assumed for body circumferences of a variable curvature radius. Fibers \& Textile in Eastern Europe, 20(6A(95)), 98102.

[21] Kowalski, K., Mielicka, E., Jasinska, I. (2012). Modelling an analysis of the circumferential force and susceptibility of vascular prostheses to internal pressure changes. Fibres \& Textile in Eastern Europe, 20(3(92)), 87-91.

[22] Leung, W. Y., Yuen, D., Ng, S. P., Shi, S. (2010). Pressure prediction model for compression garment design. Journal of Burn Care \& Research, 31, 716-727. 
AUTEX Research Journal, Vol. 20, № 1, March 2020 DOI: 10.2478/aut-2019-0006 @ AUTEX

[23] BS EN 14704-1: Determination of the elasticity of fabrics part 1: strip tests (2005).

[24] Bansal, R. (2010). Strength of materials. New Delhi, LAXMI Publication Ltd.

[25] ASTM D 5585 - 95, Standard table of body measurements for adult female misses figure type, sizes 2-20. West Conshohocken, ASTM International. 